\title{
PRESSURE DEPENDENCE OF THE RAMAN FREQUENCIES OF AMMONIA SOLID I NEAR THE MELTING POINT
}

\section{H. YURTSEVEN}

Department of Physics, Istanbul Technical University, Maslak, Istanbul, Turkey

(Received October 9, 2000)

\begin{abstract}
We calculated in this study the Raman frequencies of some lattice modes in the melting region of ammonia solid I. The Raman frequencies of those phonon modes were obtained through the Grüneisen relation using the volume data for the ammonia solid I from the literature. Our calculated Raman frequencies require for comparison the experimental Raman frequencies measured as a function of pressure for some fixed temperatures in the ammonia solid I.
\end{abstract}

PACS numbers: $63.70 .+\mathrm{h}$

\section{Introduction}

The solid-liquid phase transition in ammonia has been the subject of interest for some years. At atmospheric pressure ammonia exhibits a phase transition from the solid I phase to the liquid phase at $T_{\mathrm{m}}=192.5 \mathrm{~K}$. This melting temperature increases as the pressure increases along the melting curve. For the pressures of above $3 \mathrm{kbar}$, there occurs another phase transition from the solid II phase to the liquid phase at the temperatures of above $220 \mathrm{~K}$, along the melting curve. The first melting curve between solid I and liquid intersects the second melting curve between solid II and liquid state at the triple point, where the phase line between solid I and solid II arises in the $P-T$ phase diagram. The position of this triple point is $T_{-\mathrm{I}-\mathrm{II}}=217.34 \mathrm{~K}(3.070 \mathrm{kbar})$. The triple point between gaseous, solid I, and liquid phases is located at $T_{\mathrm{g}-\mathrm{l}-\mathrm{I}}=195.48 \mathrm{~K}(P=0)$. All these phases have been shown in the $P-T[1,2]$ and $V-T[3]$ phase diagrams in the literature. Apart from the solid I and solid II phases, it has been observed experimentally [2] that there also exists solid III (phase III) in ammonia at $35 \mathrm{kbar}$ at $25^{\circ} \mathrm{C}$.

The ammonia system has been studied as showing the critical transition near the melting point $[4,5]$. It has been stated that the ammonia undergoes a second-order phase transition prior to melting, because of the power-law behaviour of the isothermal compressibility and thermal expansivity $[4,5]$. Very recently, we have studied the critical behaviour for ammonia solid I and solid II [6]. We have then applied the Pippard relations to ammonia solid I and solid II [7]. 
In the ammonia solid I there are four translational modes of $A, E$, and $2 F$, and five rotational modes of $A, E$, and $3 F$, as the zone-centre lattice modes. They are all Raman active, as pointed out by Nye and Medina [3]. Among those lattice modes, the $A$ and $E$ modes are due to octupolar interactions according to the lattice dynamical model assuming atom-atom interactions plus electrostatic multipole interactions between molecules [8].

In this study we concentrate on the ammonia solid I considering some lattice modes studied in the work of Nye and Medina [3]. We calculate the Raman frequencies of two translational modes $\left(\approx 100 \mathrm{~cm}^{-1}\right.$ and $\left.\approx 130 \mathrm{~cm}^{-1}\right)$ and of one librational mode $\left(\approx 280 \mathrm{~cm}^{-1}\right)$ in ammonia solid I, which have been measured experimentally [3]. The Raman frequencies are calculated as a function of pressure at some fixed temperatures using our volume values calculated in our recent study [6], which were based on the work of Pruzan et al. [4]. For calculation of the Raman frequencies we also use the volume data obtained experimentally [3]. Thus, by means of the Grüneisen relation, we have been able to calculate the Raman frequencies as a function of pressure for some fixed temperatures in ammonia solid I near the melting point.

In Sec. 2 we give the theoretical basis for our calculations. In Sec. 3 we present our calculations and results for the Raman frequencies. In Secs. 4 and 5 we give discussion and conclusions, respectively.

\section{Theory}

The thermodynamic functions such as the isothermal compressibility, thermal expansivity, and the specific heat can exhibit the critical behaviour near the melting point in ammonia.

The critical behaviour of the isothermal compressibility along the pressure line near the melting point $P_{\mathrm{m}}$ in the solid ammonia, can be given by

$$
\kappa_{T}=k\left(P-P_{\mathrm{m}}\right)^{-\gamma}
$$

as given in the work of Pruzan et al. [4]. Here $\gamma$ is the critical exponent and $k$ is the amplitude. Starting from this power law expression for the isothermal compressibility $\kappa_{T}$, we can obtain the pressure dependence of solid volume $V_{\mathrm{s}}$ for the solid ammonia as

$$
V_{\mathrm{s}}=V_{\mathrm{c}} \exp \left[-k(1-\gamma)^{-1}\left(P-P_{\mathrm{m}}\right)^{1-\gamma}\right]
$$

Here $V_{c}$ represents the critical volume of the solid ammonia along the pressure line.

The volume dependence of the Raman frequencies can be obtained by means of the mode Grüneisen parameter defined as

$$
\gamma_{T}=\frac{V}{\nu}\left(\frac{\partial \nu}{\partial V}\right)_{T} .
$$

$\gamma_{T}$ is the isothermal mode Grüneisen parameter. By knowing the pressure dependence of the volume, we can calculate the Raman frequencies as a function pressure at fixed temperatures. Therefore, using the data for the solid volume $V_{\mathrm{s}}$ by means 
of Eq. (2.2) we can calculate the frequencies of some Raman modes near the melting point in ammonia. For this calculation we assume that the isothermal mode Grüneisen parameter $\gamma_{T}$ remains constant right through the phase transitions. This assumption has been considered in our earlier studies [9, 10].

In order to solve the Raman frequency as a function of pressure in terms of the volume, Eq. (2.3) can be written as

$$
\gamma_{T}=\frac{1}{\kappa_{T}} \frac{1}{\nu}\left(\frac{\partial \nu}{\partial P}\right)_{T},
$$

where the isothermal compressibility is defined as $\kappa_{T} \equiv \frac{1}{V}\left(\frac{\partial V}{\partial P}\right)_{T}$. By taking the mode Grüneisen parameter $\gamma_{T}$ as constant, from Eq. (2.4) the Raman frequency $\nu_{T}(P)$ can be obtained as

$$
\nu_{T}(P)=\Delta_{T}+A(T)+\nu_{1} \exp \left[-\gamma_{T} \ln \left(V_{T}(P) / V_{1}\right)\right]
$$

where the temperature dependent term is

$$
A(T)=a_{0}+a_{1}\left(T_{1}-T\right)+a_{2}\left(T_{1}-T\right)^{2} .
$$

In Eq. (2.6) $T_{1}$ is a given temperature, and $a_{0}, a_{1}$, and $a_{2}$ are constants. In Eq. (2.5) $\nu_{1}$ and $V_{1}$ are the values of the Raman frequency and volume, respectively, at the temperature $T_{1} . \Delta_{T}$ is the order-disorder contribution to the Raman frequency near the melting point in ammonia. We take $\Delta_{T}=0$ for $P<P_{\mathrm{c}}$ and $\Delta_{T} \neq 0$ for $P>P_{c}$. Equation $(2.5)$ has also been introduced in our earlier studies $[9,10]$.

\section{Calculations and results}

In this study we first calculated the solid volume $V_{\mathrm{s}}$ as a function of pressure by means of Eq. (2.2). For calculating the critical volume $V_{\mathrm{c}}$ in this equation we used the empirical relation for the ammonia solid I

$$
V_{\mathrm{c}}(\mathrm{I})=V_{\mathrm{I}}-d_{\mathrm{I}}\left(T-T_{\mathrm{g}-\mathrm{l}-\mathrm{I}}\right)
$$

as given by Pruzan et al. [4]. In Eq. (3.1) the values of the parameters were taken [4] as $V_{\mathrm{I}}=21.61 \mathrm{~cm}^{3} / \mathrm{mol}, d_{\mathrm{I}}=0.0316 \mathrm{~cm}^{3} /(\mathrm{mol} \cdot \mathrm{K})$ and the temperature of the triple point, $T_{\mathrm{g}-\mathrm{l}-\mathrm{I}}=195.48 \mathrm{~K}$. This gave us the temperature dependence of the critical volume for the ammonia solid $\mathrm{I}$ as

$$
V_{\mathrm{c}}(\mathrm{I})=27.79-0.0316 T \text {. }
$$

We also used the empirical relation for the melting pressure as given in the work of Pruzan et al. [4]

$$
\left.P_{\mathrm{m}}(\mathrm{I})=5.886\left[T_{\mathrm{m}} / T_{\mathrm{g}-\mathrm{l}-\mathrm{I}}\right)^{3.960}-1\right] .
$$

We then determined the values of $V_{\mathrm{c}}(\mathrm{I})$ and of $P_{\mathrm{m}}(\mathrm{I})$ by means of Eqs. (3.2) and (3.3), respectively, by taking $T=T_{\mathrm{m}}(P)$ for various temperatures ranging from $196 \mathrm{~K}$ to $217 \mathrm{~K}$ for the ammonia solid I. Table I gives our calculated $V_{\mathrm{C}}(\mathrm{I})$ and $P_{\mathrm{m}}(\mathrm{I})$ values. Using the values of the critical exponent $\gamma=0.49$ and the amplitude $k=0.0317$ for the ammonia solid I [1], we then obtained the values of the solid volume $V_{\mathrm{s}}$ as a function of the reduced pressure $P-P_{\mathrm{m}}$. We chose here the range of the reduced pressure from $0.15 \mathrm{kbar}$ to $1.8 \mathrm{kbar}$ for the ammonia solid I. 
In order to calculate the Raman frequencies as a function of the pressure, we used Eq. (2.5) where we first determined the coefficients $a_{0}, a_{1}$, and $a_{2}$ (Eq. (2.6)). For determining those coefficients we used the measured data of molar volume and the data of the lattice frequencies for two translational and one librational Raman modes due to Nye and Medina [3]. Table II gives the measured Raman frequencies for two translational modes $\left(\approx 100 \mathrm{~cm}^{-1}\right.$ and $\left.\approx 130 \mathrm{~cm}^{-1}\right)$ and for the librational mode $\left(\approx 280 \mathrm{~cm}^{-1}\right)$ with the volume data as a function of temperature, which we used for our analysis.

\section{TABLE I}

Calculated values for the critical volume $V_{\mathrm{c}}(\mathrm{I})$ and for the melting pressure $P_{\mathrm{m}}(\mathrm{I})$ by means of Eqs. (3.2) and (3.3), respectively for the ammonia solid I.

\begin{tabular}{c|c|c}
\hline \hline$T[\mathrm{~K}]$ & $V_{c}(\mathrm{I})\left[\mathrm{cm}^{3} / \mathrm{mol}\right]$ & $P_{\mathrm{m}}(\mathrm{I})[\mathrm{kbar}]$ \\
\hline 196 & 21.60 & 0.06 \\
200 & 21.47 & 0.56 \\
210 & 21.15 & 1.93 \\
215 & 21.00 & 2.70 \\
217 & 20.93 & 3.02
\end{tabular}

TABLE II

The measured Raman frequencies for two translational and one librational modes with the volume $V_{\mathrm{s}}$ data as a function of temperature for the ammonia solid I due to Nye and Medina [4].

\begin{tabular}{c|c|c|c|c}
\hline \hline$T[\mathrm{~K}]$ & $\nu\left[\mathrm{cm}^{-1}\right]$ & $\nu\left[\mathrm{cm}^{-1}\right]$ & $\nu\left[\mathrm{cm}^{-1}\right]$ & $V_{\mathrm{s}}(\mathrm{I})\left[\mathrm{cm}^{3} / \mathrm{mol}\right]$ \\
\hline 195.0 & 98.5 & 128.4 & 276.4 & 20.62 \\
187.6 & 99.1 & 129.0 & 278.2 & 20.58 \\
180.0 & 99.6 & 129.6 & 279.9 & 20.55 \\
172.8 & 100.2 & 130.2 & 281.6 & 20.52
\end{tabular}

For the translational mode of $\nu \approx 100 \mathrm{~cm}^{-1}$ we took the extrapolated values of $\nu_{1}=98.47 \mathrm{~cm}^{-1}$ and $V_{1}=21.61 \mathrm{~cm}^{3} / \mathrm{mol}$ at $T_{1}=195.48 \mathrm{~K}$, and the value of the isothermal mode Grüneisen parameter $\gamma_{T}=2.6$ [3]. Using the measured Raman frequencies $\left(\nu \approx 100 \mathrm{~cm}^{-1}\right)$ and the volume data given for four different temperatures (Table II), we were able to calculate the coefficients $\Delta_{T}+a_{0}, a_{1}$, and $a_{2}$ by means of Eq. (2.5). Those calculated values are given in Table III.

For the translational mode of $\nu \approx 130 \mathrm{~cm}^{-1}$ we took the extrapolated values of $\nu_{1}=128.36 \mathrm{~cm}^{-1}$ and $V_{1}=21.61 \mathrm{~cm}^{3} / \mathrm{mol}$ at $T_{1}=195.48 \mathrm{~K}$, and the value of $\gamma_{T}=2.35$ [3]. Similarly, using the measured Raman frequencies $\left(\nu \approx 130 \mathrm{~cm}^{-1}\right)$ 
TABLE III

Our calculated coefficients $\Delta_{T}+a_{0}, a_{1}$, and $a_{2}$ according to Eqs. (2.5) and (2.6) in the ammonia solid I. $\nu_{1}$ values are those extrapolated at $T_{1}=195.48 \mathrm{~K}$ and the $\gamma_{T}$ values were taken from Nye and Medina [4].

\begin{tabular}{c|c|c|c|c|c}
\hline \hline$\nu\left[\mathrm{cm}^{-1}\right]$ & $\nu_{1}\left[\mathrm{~cm}^{-1}\right]$ & $\gamma_{T}$ & $\begin{array}{c}\Delta_{T}+a_{0} \\
{\left[\mathrm{~cm}^{-1}\right]}\end{array}$ & $\begin{array}{c}a_{1} / \mathrm{K} \\
{\left[\mathrm{cm}^{-1}\right]}\end{array}$ & $\begin{array}{c}a_{2} \times 10^{-3} \\
{\left[\mathrm{~cm}^{-1} / \mathrm{K}^{2}\right]}\end{array}$ \\
\hline$\approx 100$ & 98.47 & 2.6 & -13.3028 & 0.0899 & -1.7 \\
$\approx 130$ & 128.36 & 2.35 & -15.269 & 0.0365 & 5.8397 \\
$\approx 280$ & 276.31 & 0.8 & -10.5316 & 0.125 & 7.116
\end{tabular}

and the volume data (Table II), we calculated $\Delta_{T}+a_{0}, a_{1}$, and $a_{2}$ through Eq. (2.5). Our calculated values are given in Table III.

For the librational mode of $\nu \approx 280 \mathrm{~cm}^{-1}$ we had the extrapolated values of $\nu_{1}=276.31 \mathrm{~cm}^{-1}$ and $V_{1}=21.61 \mathrm{~cm}^{3} / \mathrm{mol}$ at $T_{1}=195.48 \mathrm{~K}$, and the value of $\gamma_{T}=0.8$ [3]. In order to calculate the coefficients $\Delta_{T}+a_{0}, a_{1}$, and $a_{2}$ by means of Eq. (2.5), we used the measured Raman frequencies $\left(\nu \approx 280 \mathrm{~cm}^{-1}\right)$ and the volume data given in Table II. Our calculated coefficients are given in Table III.

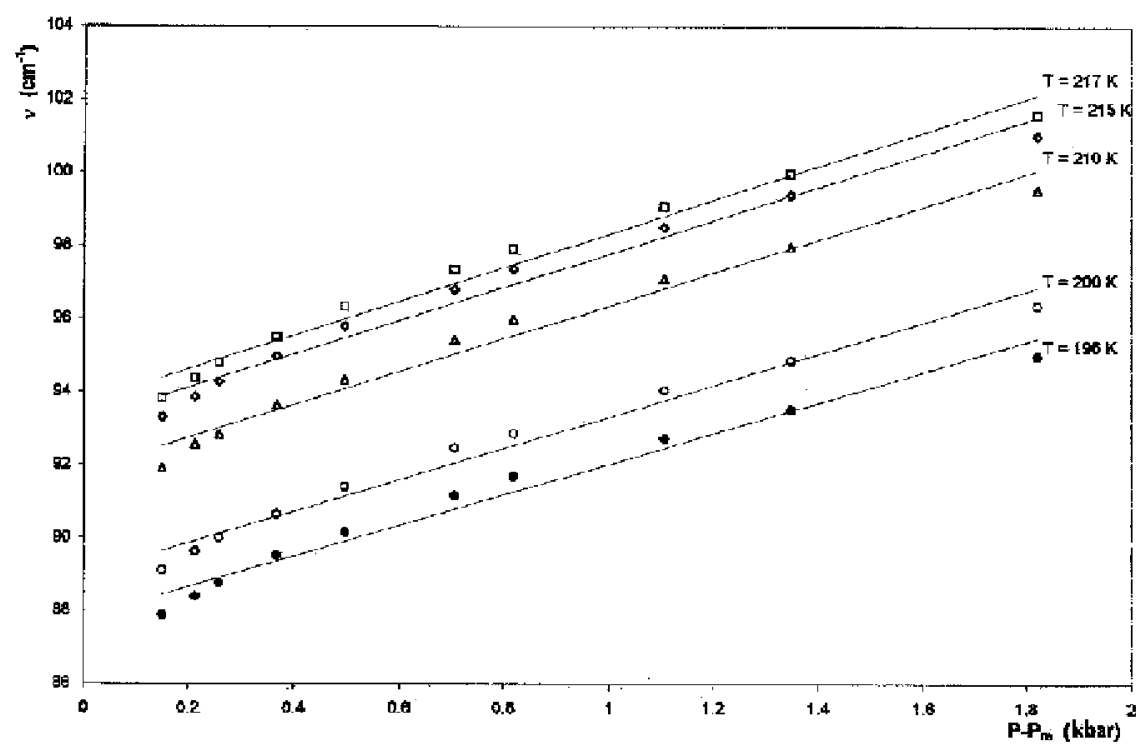

Fig. 1. Our calculated Raman frequencies as a function of the reduced pressure $P-P_{\mathrm{m}}$, where $P_{\mathrm{m}}$ is the melting pressure, for the translational mode of ammonia solid I for some fixed temperatures ranging from $196 \mathrm{~K}$ up to $217 \mathrm{~K}$. Our calculated frequencies are fitted to a straight line. 


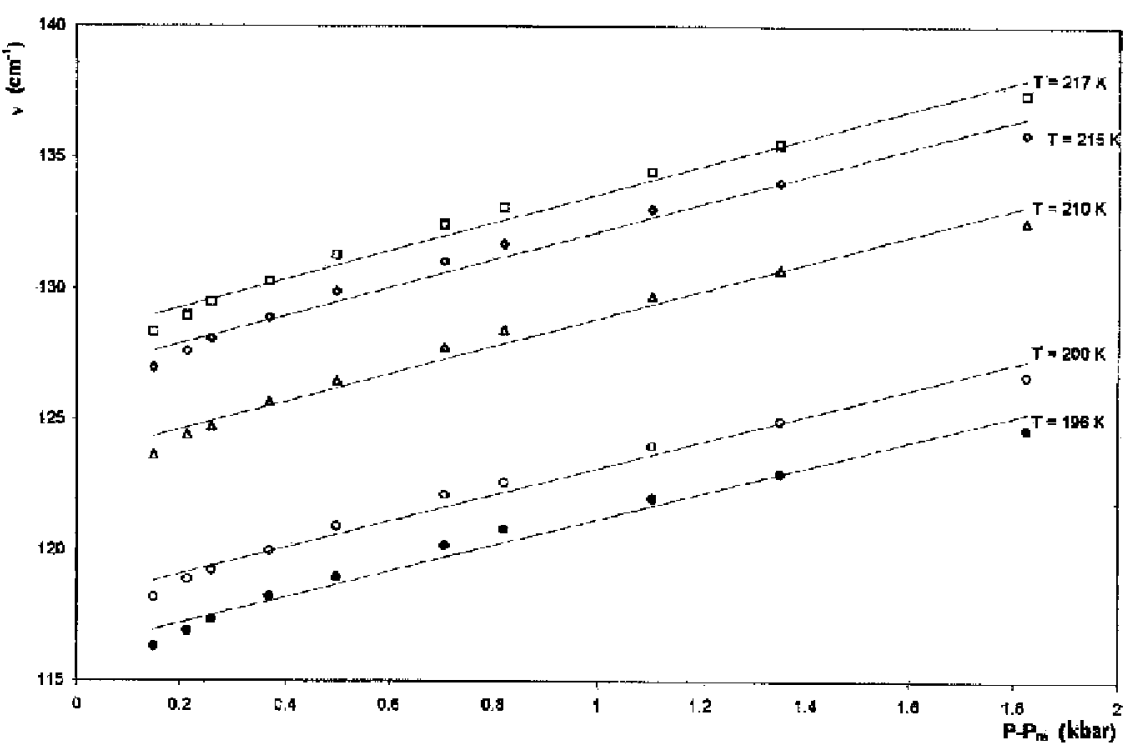

Fig. 2. Our calculated Raman frequencies as a function of the reduced pressure $P-P_{\mathrm{m}}$, where $P_{\mathrm{m}}$ is the melting pressure, for the translational mode of ammonia solid I for some fixed temperatures ranging from $196 \mathrm{~K}$ up to $217 \mathrm{~K}$. Our calculated frequencies are fitted to a straight line.

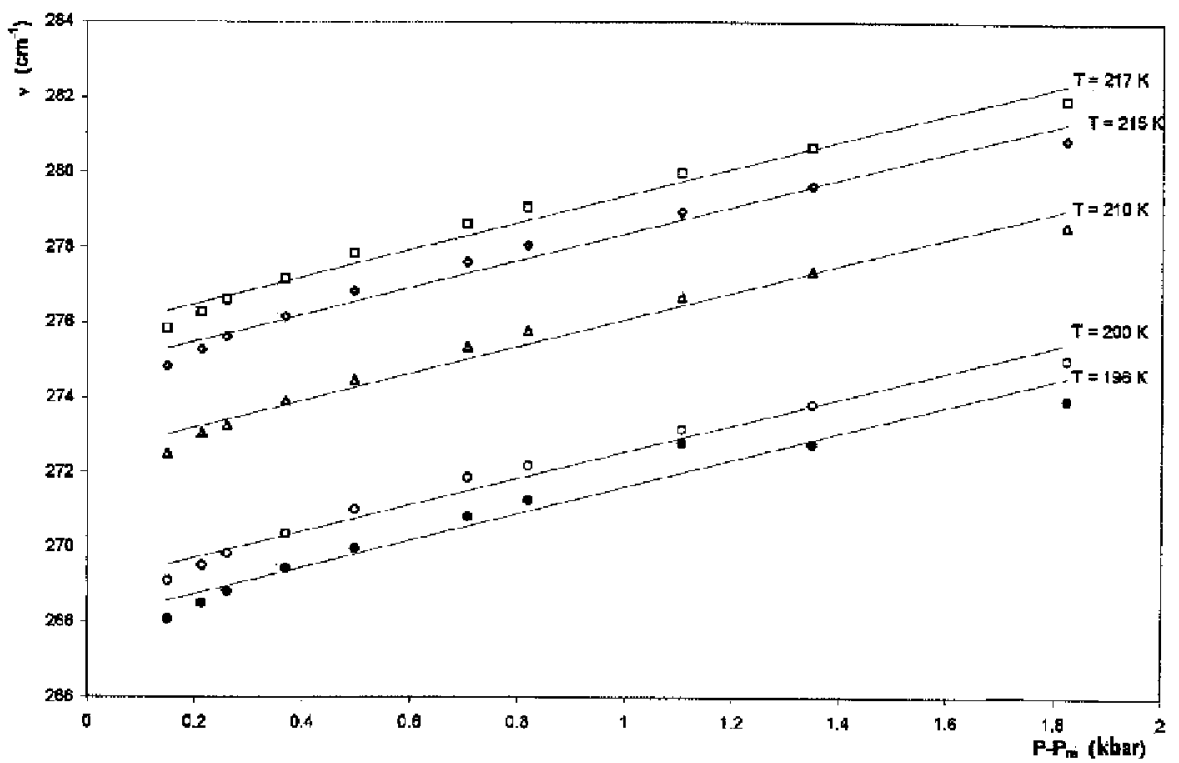

Fig. 3. Our calculated Raman frequencies as a function of the reduced pressure $P-P_{\mathrm{m}}$, where $P_{\mathrm{m}}$ is the melting pressure, for the translational mode of ammonia solid I for some fixed temperatures ranging from $196 \mathrm{~K}$ up to $217 \mathrm{~K}$. Our calculated frequencies are fitted to a straight line. 
Since we determined all the coefficients we needed, as given in Table III, we were then able to calculate the Raman frequencies as a function of pressure at some fixed temperatures by means of Eq. (2.5) for the ammonia solid I. For the frequency values calculated we used the $V_{\mathrm{s}}$ values which we determined through Eq. (2.2). For those fixed temperatures between $196 \mathrm{~K}$ and $217 \mathrm{~K}$ our calculated Raman frequencies as a function of the reduced pressure $P-P_{\mathrm{m}}$ for the translational modes of $\approx 100 \mathrm{~cm}^{-1}$ and $\approx 130 \mathrm{~cm}^{-1}$ and for the librational mode of $\approx 280 \mathrm{~cm}^{-1}$, are plotted in Figs. 1-3, respectively.

\section{Discussion}

We calculated in this study the Raman frequencies as a function of pressure for two translational modes and one librational mode of ammonia solid I. As seen from Figs. 1-3, the Raman frequencies increase as a function of pressure, as the temperature increases from $196 \mathrm{~K}$ up to $217 \mathrm{~K}$ for the translational modes of $\approx 100 \mathrm{~cm}^{-1}$ and $\approx 130 \mathrm{~cm}^{-1}$ (Figs. 1 and 2 ) and also for the librational mode of $\approx 280 \mathrm{~cm}^{-1}$ (Fig. 3). This is consistent with the decrease in the solid volume $V_{\mathrm{s}}$ as a function of pressure, as the temperature increases from $196 \mathrm{~K}$ up to $217 \mathrm{~K}$. This can also be seen partly for those values of the solid volume $V_{\mathrm{s}}(\mathrm{I})$ and for the values of the critical volume $V_{c}(\mathrm{I})$, as given in Tables $\mathrm{I}$ and II, respectively.

In calculating the Raman frequencies for the phonon modes considered here we assumed that the isothermal mode Grüneisen parameter remained constant across the melting region in the ammonia solid $I$. We took the average values of $\gamma_{T}=2.6$ and $\gamma_{T}=2.35$ for the translational modes of $\approx 100 \mathrm{~cm}^{-1}$ and $\approx 130 \mathrm{~cm}^{-1}$, respectively, as given in the work of Nye and Medina [3]. Those values of $\gamma_{T}$ are close to each other, which were determined between $172.8 \mathrm{~K}$ and $195 \mathrm{~K}$ [3]. They can also be compared with our values of 2.5 for $\nu_{5}\left(174 \mathrm{~cm}^{-1}\right)$ Raman mode of $\mathrm{NH}_{4} \mathrm{Cl}[9]$ and of 1.59 for the $\nu_{5}\left(134 \mathrm{~cm}^{-1}\right)$ Raman mode of $\mathrm{NH}_{4} \mathrm{Br}$ [11]. This indicates that regarding the values of the isothermal mode Grüneisen parameters, the phase transition of the ammonia solid I to the liquid state can be classified as the $\lambda$-type phase transition that the ammonium halides exhibit. On the other hand, the value of $\gamma_{T}=0.8$ for the librational mode of $\approx 280 \mathrm{~cm}^{-1}$ is small as compared to those values for two translational modes in ammonia solid I, as also pointed out in the work of Nye and Medina [3].

In order to compare our Raman frequencies calculated as a function of the pressure for the translational modes of $\approx 100 \mathrm{~cm}^{-1}$ and $\approx 130 \mathrm{~cm}^{-1}$ and also for the librational mode of $\approx 280 \mathrm{~cm}^{-1}$, we require the observed Raman frequencies measured as a function of pressure for the phonon modes considered in ammonia solid I. In particular, the Raman frequencies are needed at various reduced pressures ranging from $0.15 \mathrm{kbar}$ to $1.8 \mathrm{kbar}$ for fixed temperatures of $196 \mathrm{~K}$ up to $217 \mathrm{~K}$ in the melting region of the ammonia solid $\mathrm{I}$.

\section{Conclusions}

We calculated here the Raman frequencies of two translational modes and one librational mode at various pressures near the melting point for some fixed 
temperatures in the ammonia solid I. This calculation was performed by means of the Grüneisen relation using the volume data from the literature. Our calculated frequencies shift systematically as the temperature increases, as expected. The validity of our method for calculating the Raman frequencies requires the experimental measurements for the Raman frequencies as a function of pressure at fixed temperatures of the phonon modes studied here in the ammonia solid I.

\section{References}

[1] R.C. Hanson, M. Jordan, J. Phys. Chem. 84, 1173 (1980).

[2] R.L. Mills, D.H. Liebenberg, Ph. Pruzan, J. Phys. Chem. 86, 5219 (1982).

[3] C.L. Nye, F.D. Medina, Phys. Rev. B 32, 2510 (1985).

[4] Ph. Pruzan, D.H. Liebenberg, R.L. Mills, Phys. Rev. Lett. 48, 1200 (1982).

[5] Ph. Pruzan, D.H. Liebenberg, R.L. Mills, J. Phys. Chem. Solids 47, 949 (1986).

[6] H. Yurtseven, J. Phys. Chem. A 103, 5900 (1999); S. Salihoğlu, Ö. Tarı, H. Yurtseven, Phase Transit. 72, 299 (2000).

[7] H. Yurtseven, Int. J. Mod. Phys. B 13, 2783 (1999).

[8] N. Neto, R. Righini, S. Califano, S.H. Walmsley, Chem. Phys. 29, 1967 (1978).

[9] H. Yurtseven, W.F. Sherman, Phase Transit. 54, 1 (1995).

[10] H. Yurtseven, T. Demir, J. Mol. Struct. 382, 57 (1996).

[11] H. Yurtseven, M. Gülec, W.F. Sherman, Phase Transit. 56, 137 (1996). 